Research Article

\title{
Second-Order Regularity Estimates for Singular Schrödinger Equations on Convex Domains
}

\author{
Xiangxing Tao \\ Department of Mathematics, Zhejiang University of Science and Technology, Hangzhou 310023, China \\ Correspondence should be addressed to Xiangxing Tao; xxtao@hotmail.com
}

Received 21 November 2013; Accepted 17 January 2014; Published 3 March 2014

Academic Editor: Chong Li

Copyright (C) 2014 Xiangxing Tao. This is an open access article distributed under the Creative Commons Attribution License, which permits unrestricted use, distribution, and reproduction in any medium, provided the original work is properly cited.

Let $\Omega \subset \mathbb{R}^{n}$ be a nonsmooth convex domain and let $f$ be a distribution in the atomic Hardy space $H_{a t}^{p}(\Omega)$; we study the Schrödinger equations $-\operatorname{div}(A \nabla u)+V u=f$ in $\Omega$ with the singular potential $V$ and the nonsmooth coefficient matrix $A$. We will show the existence of the Green function and establish the $L^{p}$ integrability of the second-order derivative of the solution to the Schrödinger equation on $\Omega$ with the Dirichlet boundary condition for $n /(n+1)<p \leq 2$. Some fundamental pointwise estimates for the Green function are also given.

\section{Introduction and Main Results}

The regularity theory is fundamental to the partial differential equation in nonsmooth domain. Usually, the $L^{p}$ estimate of the second-order derivative of the weak solution required the smoothness of the coefficients and the smoothness of the domain. Early in 1951, Ladyzhenskaya [1] found a solution to the problem of describing the domain of the closure in $L^{2}(\Omega)$ of an elliptic operator $\mathscr{L}$ with the Dirichlet boundary condition. The solvability of the problems is based on a priori estimate,

$$
\|u\|_{W^{2,2}(\Omega)} \leq C(\Omega)\left(\|\mathscr{L} u\|_{L^{2}(\Omega)}+\|u\|_{L^{2}(\Omega)}\right) ;
$$

here $\mathscr{L}$ is a second-order elliptic operator with smooth coefficients, $\Omega$ is a bounded domain in $\mathbb{R}^{n}$ with smooth boundary, and $u$ is a function in $W^{2,2}(\Omega)$ that vanishes on the boundary or satisfies a nondegenerate homogeneous boundary condition of the first order. The significance of this result for the theory of differential operators, including the boundary value problem and the spectral theory, can hardly be overestimated.

It's certainly valuable and challenging to deduce the regularity estimate (1) for elliptic operators with rough coefficients in nonsmooth domain. In 1964, Kadlec [2] took use of the geometric properties of the convex domain to show that if $\Omega$ is a bounded convex domain in $\mathbb{R}^{n}, n>2$, and $f \in L^{2}(\Omega)$, then there is a unique solution $u \in W_{0}^{1,2}(\Omega)$ solving the Laplace equation $-\Delta u=f$ in $\Omega$, and further $\left\|\nabla^{2} u\right\|_{L^{2}(\Omega)} \leq C\|f\|_{L^{2}(\Omega)}$. In 1993, Adolfsson [3] extended Kadlec's results to get the $L^{p}$ integrability of $\nabla^{2} u$ whenever $f \in L^{p}(\Omega)$ for $1<p \leq 2$.

In the present paper, let $\Omega$ be a bounded or unbounded convex domain in $\mathbb{R}^{n}, n>2$, we consider the following singular Schrödinger operator:

$$
\mathscr{L} u:=-\operatorname{div}(A \nabla u)+V u,
$$

in $\Omega$, where $V$ is a nonnegative singular potential belonging to the class $\mathscr{B}_{q}$ for some $q \geq n / 2$, and $A(x)=\left(a^{i j}(x)\right)$ is a real symmetric matrix. We call that the potential $V$ satisfies the reverse Hölder class $\mathscr{B}_{q}$ for $1<q<\infty$, if $V \geq 0$ belongs to $L_{\text {loc }}^{q}\left(\mathbb{R}^{n}\right)$ and there exists a positive constant $K_{q}$ such that

$$
\left(\frac{1}{|B|} \int_{B} V(x)^{q} d x\right)^{1 / q} \leq \frac{K_{q}}{|B|} \int_{B} V(x) d x
$$

for all balls $B \subset \mathbb{R}^{n}$. One sees that $\mathscr{B}_{p} \subseteq \mathscr{B}_{q}$ for $1<q \leq p \leq$ $\infty$.

Recently, the regularity for the Schrödinger operator $H u=:-\Delta u+V u$ in $\mathbb{R}^{n}$, rather than in domain $\Omega$, has been studied in $[4,5]$. Shen [4] proved that if $V$ belongs to the reverse Hölder class $\mathscr{B}_{n / 2}$, then $\nabla^{2}(-\Delta+V)^{-1}$ is a CalderónZygmund operator in $\mathbb{R}^{n}$, which means that if $u$ is the solution 
to $H u=f$ in $\mathbb{R}^{n}$, then $\left\|\nabla^{2} u\right\|_{L^{p}\left(\mathbb{R}^{n}\right)} \leq C\|f\|_{L^{p}\left(\mathbb{R}^{n}\right)}$ for $1<$ $p<\infty$. We also remark that Ladyzhenskaya, see Theorem III.9.1 in [6], had found the estimate $\left\|\nabla^{2} u\right\|_{L^{2}(\Omega)} \leq C\|f\|_{L^{2}(\Omega)}$ if $\Omega$ is a bounded convex domain and $V \in L^{q}(\Omega)$ for some $q>\max (2, n / 2)$.

To discuss the singular Schrödinger equation $\mathscr{L} u=f$, we need to introduce the following assumptions (A1) and (A2) for the matrix $A(x)$ :

(A1) there exists a constant $\lambda>1$ such that

$$
\lambda^{-1}|\xi|^{2} \leq a^{i j}(x) \xi_{i} \xi_{j} \leq \lambda|\xi|^{2}, \quad \text { for any } x \in \Omega, \xi \in \mathbb{R}^{n}
$$

(A2) there exists a positive constant $M$ such that

$$
\begin{gathered}
\left|a^{i j}(x)-a^{i j}(y)\right| \leq M|x-y|, \quad \text { for any } x, y \in \Omega, \\
\qquad i, j=1,2, \ldots, n, \\
\sum_{i=1}^{n} \frac{\partial a^{i j}(x)}{\partial x_{i}}=0 \quad \forall j=1,2, \ldots, n .
\end{gathered}
$$

The last assumption above in (A2) means that we can rewrite the operator as $\mathscr{L} u(x)=-\sum_{i, j} a^{i j}(x)\left(\partial^{2} u(x) /\right.$ $\left.\partial x_{i} \partial x_{j}\right)+V(x) u(x)$. In the case $\Omega=\mathbb{R}^{n}$, by the CalderónZygmund singular integral theory, Avellaneda and Lin [7] showed the $L^{p}\left(\mathbb{R}^{n}\right)$-boundedness of the operator $\nabla^{2} \mathscr{L}^{-1}$ for $1<p<\infty$ under assumptions (A1), (A2), and

(A3) there is $0<\alpha \leq 1$ such that

$a^{i j} \in C^{1+\alpha}, \quad a^{i j}(x)=a^{i j}(x+z)$ for any $x \in \mathbb{R}^{n}, z \in \mathbb{Z}^{n}$.

Kurata and Sugano [8] also obtained the weighted $L^{p}\left(\mathbb{R}^{n}\right)$ boundedness of the operator $\nabla^{2} \mathscr{L}^{-1}$ for $1<p<\infty$ under the assumptions (A1), (A2), and (A3).

Here we remark that for general convex domain $\Omega$ and the nonsmooth coefficient matrix $A(x)$, the associated operator $\nabla^{2} \mathscr{L}^{-1}$ is not always a Calderón-Zygmund operator, and the methods used in $[4,5,7,8]$ cannot be applied to these cases.

The purpose of the paper is to give an elemental proof of the $L^{p}(\Omega)$ boundedness and the $H^{p}(\Omega)$ boundedness for the operator $\nabla^{2} \mathscr{L}^{-1}$ on the convex domain $\Omega \subset \mathbb{R}^{n}$ without assumption (A3). Equivalently, we will study the existence and the regularity of the weak solution $u=\mathscr{L}^{-1} f$ to the following Dirichlet problem in the convex domain $\Omega$, that is

$$
\mathscr{L} u=f \quad \text { in } \Omega, \quad \gamma u=0 \quad \text { on } \partial \Omega,
$$

for $f \in H_{a t}^{p}(\Omega)$ with $n /(n+1)<p \leq 1$, the atomic Hardy spaces, or $f \in L^{p}(\Omega)$ with $1<p \leq 2$, where $\gamma$ is the trace operator on the boundary $\partial \Omega$ of the domain $\Omega$.

For our purpose, let $k$ be the integer and let $0<p<\infty$, we denote by

$$
W^{k, p}(\Omega)=\left\{f \in L^{p}(\Omega): \sum_{l=0}^{k}\left(\int_{\Omega}\left|\nabla^{l} f(x)\right|^{p} d x\right)^{1 / p}<\infty\right\}
$$

the Sobolev spaces, and denote by $W_{0}^{k, p}(\Omega)$ the closure of $C_{0}^{\infty}(\Omega)$ in $W^{k, p}(\Omega)$.

We call $a(x)$ a $p$-atom, if $a(x)$ is a bounded measurable function defined in $\Omega$ and the following conditions (i), (ii), and (iii) hold:

(i) there is a cube $Q \subset \Omega$ satisfying supp $a(x) \subset Q$;

(ii) $\|a\|_{L^{2}\left(\mathbb{R}^{n}\right)} \leq|Q|^{1 / 2-1 / p}$;

(iii) $\int x^{\beta} a(x) d x=0$ for any multi-index $\beta$ with $|\beta| \leq$ $[n / p-n]$.

The atomic Hardy space in domain $\Omega, H_{a t}^{p}(\Omega)$, is then defined as the collection of all $f=\sum \lambda_{j} a_{j}$ in the sense of distributions, where $\left\{a_{j}\right\}$ is a sequence of $p$-atoms and $\left\{\lambda_{j}\right\}$ is a sequence of real numbers with $\sum\left|\lambda_{j}\right|^{p}<\infty$. The norm of $f$ is defined by

$$
\|f\|_{H_{a t}^{p}(\Omega)}=\inf \left\{\left(\sum\left|\lambda_{j}\right|^{p}\right)^{1 / p}: f=\sum \lambda_{j} a_{j}\right\} .
$$

One might see [9] for $H^{p}$ space over open subsets in $\mathbb{R}^{n}$. It's worthy to point out that if $\Omega$ is a Lipschitz domain or a convex domain then we can see from the works in $[10,11]$ that $H_{a t}^{p}(\Omega)=H_{z}^{p}(\Omega)$, where $H_{z}^{p}(\Omega)$ is the following local Hardy space in domain,

$$
\begin{gathered}
H_{z}^{p}(\Omega)=\left\{f \in \mathcal{S}^{\prime}(\Omega): \text { there is } F \in H^{p}\left(\mathbb{R}^{n}\right)\right. \\
\text { such that } \left.\left.F\right|_{\Omega}=f,\left.F\right|_{\overline{\Omega^{c}}}=0\right\} .
\end{gathered}
$$

We also notice that the dual space of $H_{a t}^{p}(\Omega)$ with $n /(n+$ $1)<p<1$ is the space of Hölder continuous functions, $C^{\alpha(p)}(\Omega)$, with the exponent $\alpha(p)=n(1-p) / p$. Thus the paring between an element of $H_{a t}^{p}(\Omega)$ and the function in $C^{\alpha(p)}(\Omega)$ is well defined. One could refer to [12] for related $H^{p}$ boundary value problems.

For $f \in H_{a t}^{p}(\Omega), n /(n+1)<p<1$, we say $u$ is a solution to the Dirichlet problem (7), if $u \in W_{0}^{1,2}(\Omega) \cap W^{1, p}(\Omega)$ satisfies

$$
\begin{gathered}
\int_{\Omega} A(x) \nabla u(x) \nabla \psi(x) d x+\int_{\Omega} V(x) u(x) \psi(x) d x \\
=\int_{\Omega} f(x) \psi(x) d x,
\end{gathered}
$$

for any test function $\psi(x) \in C^{\alpha(p)}(\Omega) \cap W_{0}^{1,2}(\Omega)$.

Applying the Lax-Milgram theorem, we will prove that for the Lipschitz domain $\Omega$ and the function $f \in L^{2}(\Omega)$, there is a unique solution $u \in W_{0}^{1,2}(\Omega)$ to the Dirichlet problem (7); see Theorem 10 below. We will then show the existence of the Green function related to the operator $\mathscr{L}$ and the domain $\Omega$ and give the point-wise estimates for the Green function which is fundamental to us. Moreover, we will give the $L^{2}$ boundedness for the second-order derivative of the solution,

$$
\left\|\nabla^{2} u\right\|_{L^{2}(\Omega)} \leq C\|f\|_{L^{2}(\Omega)}
$$

see Theorems 20 and 21 below.

Our main aim is to further establish the second-order regularity estimates for the equation $\mathscr{L} u=f$ in $\Omega$ with $f \in H_{a t}^{p}(\Omega)$. 
Theorem 1. Suppose that $\Omega$ is a bounded convex domain, $V \in$ $\mathscr{B}_{n}$ and $A$ satisfies assumptions (A1) and (A2). If $f \in H_{a t}^{p}(\Omega)$ for $n /(n+1)<p \leq 1$ and $u \in W_{0}^{1,2}(\Omega) \cap W^{1, p}(\Omega)$ solves the Dirichlet problem (7), then one has

$$
\left\|\nabla^{2} u\right\|_{L^{p}(\Omega)} \leq C\|f\|_{H_{a t}^{p}(\Omega)},
$$

with the constant $C$ independent of $f$.

Theorem 2. Let $\Omega$ be the region above a convex Lipschitz graph, and let $V \in \mathscr{B}_{n}$. If $f \in H_{a t}^{p}(\Omega)$ for $n /(n+1)<p \leq 1$ and $u \in W_{0}^{1,2}(\Omega) \cap W^{1, p}(\Omega)$ satisfies $-\Delta u+V u=f$ in $\Omega$, then one has

$$
\left\|\nabla^{2} u\right\|_{L^{p}(\Omega)} \leq C\|f\|_{H_{a t}^{p}(\Omega)},
$$

with the constant $C$ independent of $f$.

By the interpolation argument between the $L^{2}$-estimate (12) and Theorems 1 and 2 , we have the following $L^{p}$-regularity estimates for $1<p \leq 2$.

Corollary 3. Suppose that $\Omega$ is a bounded convex domain, $V \in$ $\mathscr{B}_{n}$ and $A$ satisfies assumptions (A1) and (A2). If $f \in L^{p}(\Omega)$ for $1<p \leq 2$ and $u \in W_{0}^{1,2}(\Omega) \cap W^{1, p}(\Omega)$ solves the Dirichlet problem (7), then one has

$$
\left\|\nabla^{2} u\right\|_{L^{p}(\Omega)} \leq C\|f\|_{L^{p}(\Omega)},
$$

with the constant $C$ independent of $f$.

Corollary 4. Let $\Omega$ be the region above a convex Lipschitz graph, and let $V \in \mathscr{B}_{n}$. If $f \in L^{p}(\Omega)$ for $1<p \leq 2$ and $u \in W_{0}^{1,2}(\Omega) \cap W^{1, p}(\Omega)$ satisfies $-\Delta u+V u=f$ in $\Omega$, then one has

$$
\left\|\nabla^{2} u\right\|_{L^{p}(\Omega)} \leq C\|f\|_{L^{p}(\Omega)},
$$

with the constant $C$ independent of $f$.

Remark 5. One can see from Theorem 10 in Section 2 and the arguments for the proof of Theorems 1 and 2 that the condition $V \in \mathscr{B}_{n}$ couldn't be reduced for the second-order derivative estimates of the solution, but the condition $V \in$ $\mathscr{B}_{n / 2}$ is enough for the existence of $W_{0}^{1,2}(\Omega)$-solution to the Dirichlet problem (7).

The paper is organized in the following way. In Section 2, after recalling some properties for the class $\mathscr{B}_{n}$, we will show the solvability and uniqueness of the $W_{0}^{1,2}(\Omega)$ solution to the Dirichlet problem (7); see Theorem 10 below. We will also give some useful point-wise estimates for the Green function $G(x, y)$ and its gradient $\nabla_{x} G(x, y)$ related to the singular Schrödinger operator $\mathscr{L}$ in the convex domain $\Omega$; see Lemmas 12-16 below for details. In Section 3, we will deduce some important estimates for the solution $u$ to $\mathscr{L} u=f$ in $\Omega$, especially, the local $L^{2}$-estimates for the second-order derivative of the solution $u$; see Theorems 20 and 21 below. In Section 4, we will give the proofs of Theorems 1 and 2.

\section{The $W_{0}^{1,2}(\Omega)$ Solutions and the Green Function}

In this section, we will show the existence of the $W_{0}^{1,2}(\Omega)$ solution to the singular Schrödinger equation $\mathscr{L} u=f$ in Lipschitz domain $\Omega$ for $f \in L^{2}(\Omega)$ and give some estimates about the Green function related to the operator $\mathscr{L}$ in $\Omega$. To this end, we need to use an auxiliary function and some properties for the singular potential $V$. Let $V \in \mathscr{B}_{n / 2}$, we can define the auxiliary function $m(V, x)$ by

$$
\frac{1}{m(V, x)}=\sup _{r>0}\left\{r: \frac{1}{r^{n-2}} \int_{B(x, r)} V(y) d y \leq 1\right\} \text {. }
$$

Recall that $V \in \mathscr{B}_{n / 2}$ implies that $V(x) d x$ is a doubling measure, and $V \in \mathscr{B}_{q}$ for some $q>n / 2$. Thus, by the Hölder inequality, for any $0<r \leq R<\infty$,

$$
\int_{B(x, r)} V(y) d y \leq C\left(\frac{r}{R}\right)^{n-2+\delta} \int_{B(x, R)} V(y) d y,
$$

with some $\delta>0$. Therefore, the auxiliary function $m(V, x)$ is well defined and $0<m(V, x)<\infty$. For example, if $P(x)$ is a polynomial of degree $k$ and $V(x)=|P(x)|$, then

$$
m(V, x) \simeq \sum_{|\beta| \leq k}\left|\partial_{x}^{\beta} P(x)\right|^{1 /(|\beta|+2)}
$$

Lemma 6 (see [4]). Let $V \in \mathscr{B}_{q}$ for $q \geq n / 2$, then there exist constants $C>0, c>0$ and $k_{0}>0$ such that for any $x, y$ in $\mathbb{R}^{n}$,

(i) $m(V, x) \sim m(V, y)$, if $|x-y| \leq 1 / m(V, x)$;

(ii) $m(V, y) \leq C\{1+|x-y| m(V, x)\}^{k_{0}} m(V, x)$;

(iii) $m(V, y) \geq\left(c m(V, x) /\{1+|x-y| m(V, x)\}^{k_{0} /\left(k_{0}+1\right)}\right)$;

(iv) $c\{1+|x-y| m(V, x)\}^{1 /\left(k_{0}+1\right)} \leq 1+|x-y| m(V, y) \leq$ $C\{1+|x-y| m(V, x)\}^{k_{0}+1}$.

The estimates of (i), (ii), and (iii) in Lemma 6 were proved in [4], while the estimate (iv) can be derived from the estimates (ii) and (iii).

Lemma 7 (see [13]). Let $q>s \geq 0, q \geq \max \{1, s n / \alpha\}, \alpha>0$, and $k$ is sufficiently large, then there are positive constants $k_{0}$, $C$, and $C_{k}$ such that

$$
\begin{gathered}
\int_{|x-y|<r} \frac{V(y)^{s}}{|x-y|^{n-\alpha}} d Y \leq C r^{\alpha-2 s}\{1+r m(V, x)\}^{s k_{0}}, \\
\int_{R^{n}} \frac{V(y)^{s} d Y}{\{1+m(V, x)|x-y|\}^{k}|x-y|^{n-\alpha}} \leq C_{k} m(V, x)^{2 s-\alpha},
\end{gathered}
$$

for any $r>0, x \in \mathbb{R}^{n}$ and $V \in \mathscr{B}_{q}$.

Lemma 8 (see [8]). Let $\Gamma(x, y)$ denote the fundamental solution to equation $\mathscr{L} u=0$ in $\mathbb{R}^{n}$. Then for any integer $k>0$, one has that 
(i) if $V \in \mathscr{B}_{n / 2}$, then there exists a constant $C_{k}$ such that

$$
0 \leq \Gamma(x, y) \leq \frac{C_{k}}{\{1+|x-y| m(V, x)\}^{k}|x-y|^{n-2}},
$$

for any $x, y$ in $\mathbb{R}^{n}$;

(ii) if $V \in \mathscr{B}_{n}$, also we assume $a^{i j}(x)$ satisfies $\left\|a^{i j}(x)\right\|_{C^{\alpha}\left(R^{n}\right)} \leq C_{1}$ with constant $C_{1}>0$ and $\alpha \epsilon$ $(0,1]$, then there exists a constant $C_{k}$ such that

$$
\left|\nabla_{x} \Gamma(x, y)\right| \leq \frac{C_{k}}{\{1+|x-y| m(V, x)\}^{k}|x-y|^{n-1}},
$$

for any $x, y$ in $\mathbb{R}^{n}$.

The following lemma is useful for proving the $L^{2}$ solvability to the Dirichet problem, which extends the FeffermanPhong inequality and has been showed in [14] for the case $\Omega=\mathbb{R}^{n}$. Here we thank the referee for pointing out that Lemma 9 below can be generalized to more general domains by applying the embedding estimates in [15] among others.

Lemma 9. Let $\Omega$ be a convex domain in $\mathbb{R}^{n}$ and let $V \in \mathscr{B}_{q}$ for $q \geq n / 2$. Then, for $u \in C_{0}^{1}(\Omega)$,

$$
\begin{aligned}
& \int_{\Omega}|u(x)|^{2} m(V, x)^{2} d x \\
& \leq C\left\{\int_{\Omega}|\nabla u|^{2} d x+\int_{\Omega}|u(x)|^{2} V(x) d x\right\}, \\
& \int_{\Omega}|u(x)|^{2} V(x) d x \\
& \leq C\left\{\int_{\Omega}|\nabla u|^{2} d x+\int_{\Omega}|u(x)|^{2} m(V, x)^{2} d x\right\},
\end{aligned}
$$

with the absolute constant $C=C\left(n, \lambda, K_{q}\right)$.

Proof. Along the same lines as that in [14], we can claim the following Poincaré inequality:

$$
\begin{aligned}
& \iint_{D_{r}}|u(x)-u(y)|^{2} V(y) d y d x \\
& \quad \leq C r^{2} \int_{D_{r}} V(y) d y \int_{D_{r}}|\nabla u(x)|^{2} d x,
\end{aligned}
$$

where $D_{r}=B\left(x_{0}, r\right) \cap \Omega$ for $x_{0} \in \Omega$. The inequality (24) for case $\Omega=\mathbb{R}^{n}$ was founded in [14]; here we adapt the argument and give the simple lines of the proof for completeness. In fact, for $x, y \in D_{r}$, one notes that $D_{r}$ is a convex domain and so one can write that

$$
u(x)-u(y)=\int_{0}^{|x-y|} \nabla u\left(y+t \frac{x-y}{|x-y|}\right) \cdot \frac{x-y}{|x-y|} d t .
$$

Let $f=\nabla u$ and, for $x, y \in D_{r}$, we define

$$
\begin{array}{r}
T_{j} f(x, y)=\int_{\substack{2^{-j} r \leq t<2^{-j+1} r \\
0<|x-y|}} f\left(y+t \frac{x-y}{|x-y|}\right) d t, \\
j=0,1,2, \ldots
\end{array}
$$

It's clear that $y+t((x-y) /|x-y|) \in D_{r}$ for $0<t<|x-y|$, and so

$$
\left|T_{j} f(x, y)\right| \leq 2^{-j} r\|f\|_{L^{\infty}\left(D_{r}, d x\right)} .
$$

Also, by the Fubini theorem, the doubling property of measure $V d y$, and the inequality (18), one can deduce that (see page 527 in [14])

$$
\begin{aligned}
\iint_{D_{r}}\left|T_{j} f(x, y)\right| V(y) d y d x \\
\quad \leq C 2^{-j(\delta-1)} r \int_{B\left(x_{0}, r\right)} V(y) d y \cdot\|f\|_{L^{1}\left(D_{r}, d x\right)} .
\end{aligned}
$$

Combining the inequalities (27) and (28), we get

$$
\begin{aligned}
& \left\|T_{j} f\right\|_{L^{2}\left(D_{r} \times D_{r}, V(y) d y d x\right)} \\
& \quad \leq C(\sqrt{2})^{-j \delta} r\left(\int_{B\left(x_{0}, r\right)} V(y) d y\right)^{1 / 2} \cdot\|f\|_{L^{1}\left(D_{r}, d x\right)},
\end{aligned}
$$

by interpolation. By summation and the Minkowski inequality, we have

$$
\begin{aligned}
\iint_{D_{r}}|u(x)-u(y)|^{2} V(y) d y d x \\
\quad \leq C r^{2} \int_{B\left(x_{0}, r\right)} V(y) d y \int_{D_{r}}|\nabla u(x)|^{2} d x .
\end{aligned}
$$

Since $\Omega$ is a Lipschitz domain and $x_{0} \in \Omega$, there exists $B\left(y_{0}, c r\right) \subset D_{r}$ for some $y_{0} \in D_{r}$ and $c>0$, depending only on the Lipschitz character of $\Omega$. Thus, by the doubling property of $V d y$,

$$
\begin{aligned}
\int_{B\left(x_{0}, r\right)} V(y) d y & \leq \int_{B\left(y_{0}, 2 r\right)} V(y) d y \\
& \leq C \int_{B\left(y_{0}, c r\right)} V(y) d y \leq C \int_{D_{r}} V(y) d y .
\end{aligned}
$$

This, together with (30), implies (24).

Let $r_{0}=1 / m\left(V, x_{0}\right)$, by (31) and the definition of $m\left(V, x_{0}\right)$, one sees that

$$
\int_{D_{r_{0}}} V(y) d y \simeq \int_{B\left(x_{0}, r_{0}\right)} V(y) d y \simeq r_{0}^{n-2} .
$$

Now applying the Poincaré inequality (24), we obtain that

$$
\begin{aligned}
& \frac{1}{r_{0}^{n+2}} \int_{D_{r_{0}}}|u(x)|^{2} d x \\
& \quad \leq \frac{C}{r_{0}^{n}} \int_{D_{r_{0}}}|\nabla u(x)|^{2} d x+\frac{C}{r_{0}^{n}} \int_{D_{r_{0}}}|u(x)|^{2} V(x) d x, \\
& \frac{1}{r_{0}^{n}} \int_{D_{r_{0}}}|u(x)|^{2} V(x) d x \\
& \quad \leq \frac{C}{r_{0}^{n}} \int_{D_{r_{0}}}|\nabla u(x)|^{2} d x+\frac{C}{r_{0}^{n+2}} \int_{D_{r_{0}}}|u(x)|^{2} d x .
\end{aligned}
$$


We integrate both sides of (33) and (34), respectively, with respect to $x_{0}$ over $\Omega$. By the Fubini theorem and Lemma 6 we will obtain the inequalities (23). The lemma is proved.

Next we let $\mathscr{H}(\Omega)$ be the class of all functions $u \in W_{0}^{1,2}(\Omega)$ such that

$$
\begin{aligned}
& \|u\|_{\mathscr{H}(\Omega)} \\
& \quad:=\left(\int_{\Omega}|\nabla u(x)|^{2} d x+\int_{\Omega} m(V, x)^{2}|u(x)|^{2} d x\right)^{1 / 2}<\infty .
\end{aligned}
$$

Then $\mathscr{H}(\Omega)$ is a Hilbert space and $C_{0}^{1}(\Omega)$ is dense in $\mathscr{H}(\Omega)$. Let

$$
\begin{aligned}
a(u, v)= & \int_{\Omega} A(x) \nabla u(x) \nabla v(x) d x \\
& +\int_{\Omega} V(x) u(x) v(x) d x,
\end{aligned}
$$

then we can see from the elliptic condition of the matrix $A(x)$ and Lemma 9 that

$$
|a(u, v)| \leq C\|u\|_{\mathscr{H}(\Omega)}\|v\|_{\mathscr{H}(\Omega)},
$$

for all $u, v \in \mathscr{H}(\Omega)$; and by Lemma 9 , there is a positive constant $\delta$ independent of $u$ such that

$$
a(u, u) \geq \lambda^{-1} \int_{\Omega}|\nabla u|^{2} d x+\int_{\Omega}|u(x)|^{2} V(x) d x \geq \delta\|u\|_{\mathscr{H}(\Omega)}^{2} .
$$

Thus $a(u, v)$ is a bounded, coercive bilinear form on the Hilbert space $\mathscr{H}(\Omega)$.

On the other hand, for $f_{1} \in L^{2}(\Omega)$ and $m(V, \cdot)^{-1} f_{2} \in$ $L^{2}(\Omega)$, let $F(v)=\int_{\Omega}\left(f_{1}(x)+f_{2}(x)\right) v(x) d x$ for $v \in \mathscr{H}(\Omega)$. Then by the Hölder inequality and the Poinceré inequality one gets

$$
\begin{aligned}
|F(v)| & \leq\left\|f_{1}\right\|_{L^{2}(\Omega)}\|v\|_{L^{2}(\Omega)}+\left\|m(V, \cdot)^{-1} f_{2}\right\|_{L^{2}(\Omega)}\|m(V, \cdot) v\|_{L^{2}(\Omega)} \\
& \leq\left(C|\Omega|^{1 / n}\left\|f_{1}\right\|_{L^{2}(\Omega)}+\left\|m(V, \cdot)^{-1} f_{2}\right\|_{L^{2}(\Omega)}\right)\|v\|_{\mathscr{H}(\Omega)},
\end{aligned}
$$

which means $F \in \mathscr{H}^{*}(\Omega)$, a bounded linear functional on $\mathscr{H}(\Omega)$.

Thus using the Lax-Milgram theorem we obtain the following $L^{2}$ solvability of the Dirichlet problem (7).

Theorem 10. Suppose that $\Omega$ is a bounded convex domain, and $V \in \mathscr{B}_{q}$ for $q \geq n / 2$. Let $f_{1} \in L^{2}(\Omega)$ and let $m(V, \cdot)^{-1} f_{2} \in$ $L^{2}(\Omega)$, then there is a unique weak solution $u \in W_{0}^{1,2}(\Omega)$ solving the singular Schrödinger equation $\mathscr{L} u=f_{1}+f_{2}$ in $\Omega$, and further

$$
\begin{aligned}
\int_{\Omega} \mid \nabla & \left.u(x)\right|^{2} d x+\int_{\Omega} V(x)|u(x)|^{2} d x \\
& \leq C|\Omega|^{2 / n} \int_{\Omega}\left|f_{1}(x)\right|^{2} d x+C \int_{\Omega} m(V, x)^{-2}\left|f_{2}(x)\right|^{2} d x
\end{aligned}
$$

with the absolute constant $C=C\left(n, \lambda, K_{q}\right)$.
Proof. The estimate (40) follows from Lemma 9, the inequalities (38) and (39).

Remark 11. Checking the argument above, we note that if $f_{1}=0$ then the results of Theorem 10 are still true for any unbounded convex domain $\Omega$.

In this paper, we always let $V \in \mathscr{B}_{q}$ for some $q \geq n / 2$, thus by Theorem 10 and Remark 11, we have the Green function $G(x, y)$ defined on $\Omega \times \Omega$ for any convex domain $\Omega$ such that, for each $y \in \Omega$ and any $r>0, G(\cdot, y) \in W^{1,2}(\Omega \backslash B(y, r)) \cap$ $W_{0}^{1,1}(\Omega)$, and $\mathscr{L} G(\cdot, y)=\delta_{y}$ in the distribution sense. Noting $V \geq 0$, we know by maximal principle that for any $x, y \in \Omega$,

$$
0 \leq G(x, y)=G(y, x) \leq \frac{C}{|x-y|^{n-2}},
$$

with the constant $C=C\left(n, \lambda, K_{q}\right)$ independent of $x, y \in$ $\Omega$. Moreover, we can show the following decay estimates as Lemma 2.7 in [13] or Lemma 1.21 in [14]; here we omit the details of the proof.

Lemma 12. Let $k>0$ be any integer, then

$$
|G(x, y)| \leq \frac{C_{k}}{\{1+|x-y| m(x, V)\}^{k}} \frac{1}{|x-y|^{n-2}},
$$

where $C_{k}=C\left(k, n, \lambda, K_{q}\right)$ is the constant independent of $x, y \in$ $\Omega$.

Next in this section, we suppose that $\Omega$ is a bounded convex domain or the region above a Lipschitz graph. Noting that one may take a cone of arbitrary height and fixed opening angle at any boundary point of the Lipschitz graph, by similar argument as that of Theorems 1.8 and 1.9 in [16], we can deduce the following Hölder estimate for the Green function $G(x, y)$.

Lemma 13. Let $k>0$ be any integer, then there are constants $C=C\left(n, k, \lambda, K_{q}\right)$ and $\alpha=\alpha(n, \lambda), 0<\alpha<1$, such that for all $x, y, z \in \Omega$,

$$
\begin{aligned}
& |G(x, y)-G(z, y)| \\
& \quad \leq \frac{C}{\{1+|x-y| m(V, y)\}^{k}}\left(\frac{|x-z|^{\alpha}}{|x-y|^{n-2-\alpha}}+\frac{|x-z|^{\alpha}}{|z-y|^{n-2-\alpha}}\right) .
\end{aligned}
$$

Using the similar arguments as that of Theorems 3.3(ii) and 3.4(ii) in [16], we also have the following estimate.

Lemma 14. Let $k>0$ be any integer, then there is $C=$ $C\left(n, k, \lambda, K_{q}, M\right)>0$ such that, for all $x, y, z \in \Omega$,

$$
|G(x, y)| \leq \frac{C}{\left\{1+|x-y| m\left(x_{0}, V\right)\right\}^{k}} \frac{\delta(x)}{|x-y|^{n-1}},
$$

where $\delta(x)=\operatorname{dist}(x, \partial \Omega)$.

In order to get the derivative estimates for the Green function $G(x, y)$, we need to show the following lemma. 
Lemma 15. Suppose $V \in \mathscr{B}_{n}$. If $w \in W^{1,2}\left(B\left(x_{0}, r\right)\right)$ satisfies the equation $-\operatorname{div}(A(x) \nabla w(x))+V(x) w(x)=0$ in $B\left(x_{0}, r\right)$, then there exists a positive constant $C=C\left(n, \lambda, K_{n}, M\right)$ independent of $x_{0}$ and $r$ such that

$$
\left|\nabla w\left(x_{0}\right)\right| \leq \frac{C}{r} \sup _{x \in B\left(x_{0}, r\right)}|w(x)| .
$$

Proof. Let $\phi \in C_{0}^{\infty}\left(B\left(x_{0}, r\right)\right)$ be the cut-off function, then we have

$$
\int_{B\left(x_{0}, r\right)}\left\{A \nabla w \cdot \nabla\left(w \phi^{2}\right)+V w^{2} \phi^{2}\right\} d x=0
$$

and so

$$
\begin{aligned}
\int_{B\left(x_{0}, r\right)} & \left\{A \nabla(w \phi) \cdot \nabla(w \phi)+V w^{2} \phi^{2}\right\} d x \\
= & \int_{B\left(x_{0}, r\right)} A \nabla \phi \cdot \nabla \phi w^{2} d x,
\end{aligned}
$$

which implies the following Caccioppoli inequality:

$$
\int_{B\left(x_{0}, r_{1}\right)}\left[|\nabla w|^{2}+V|w|^{2}\right] d x \leq \frac{C \lambda^{2}}{\left(r_{2}-r_{1}\right)^{2}} \int_{B\left(x_{0}, r_{2}\right)}|w|^{2} d x,
$$

with the absolute constant $C$ independent of $r_{1}$ and $r_{2}$ with $0 \leq r_{1}<r_{2} \leq r$.

Observing that

$$
-\operatorname{div}(A \nabla(w \phi))+V w \phi=-2 A \nabla w \nabla \phi-\operatorname{div}(A \nabla \phi) w
$$

and letting the cut-off function $\phi \in C_{0}^{\infty}\left(B\left(x_{0}, r / 2\right)\right)$ such that $\phi \equiv 1$ on $B\left(x_{0}, r / 3\right)$ and $|\nabla \phi| \leq 1 / r$, then we have

$$
\begin{aligned}
\nabla w\left(x_{0}\right)=\int \nabla_{x} \Gamma\left(x_{0}, y\right)[ & -2 A(y) \nabla w(y) \nabla \phi(y) \\
& -\operatorname{div}(A(y) \nabla \phi(y)) w(y)] d y .
\end{aligned}
$$

Hence, from this, and using Lemma 8 and the Caccioppli inequality (48), we obtain the desired estimate of the lemma.

Lemma 16. Suppose $V \in \mathscr{B}_{n}$, and that $k>0$ is any integer. Then

$$
\left|\nabla_{x} G(x, y)\right| \leq \frac{C_{k}}{\{1+|x-y| m(V, x)\}^{k}} \frac{1}{|x-y|^{n-1}},
$$

where $C_{k}=C\left(n, k, \lambda, K_{n}, M\right)$ is independent of $x, y \in \Omega$.

Proof. For $x, y \in \Omega$, we let $r=|x-y|$ and denote by $\delta(x)=$ $\operatorname{dist}(x, \partial \Omega)$. If $\delta(x)>r / 2$, thus $B(x, r / 2) \subset \Omega$. One notes that
$G^{y}:=G(\cdot, y)$ satisfies $L G^{y}=0$ in $B(x, r / 2)$ for the fixed $y$. By Lemmas 12 and 15 , one can see that

$$
\begin{aligned}
\left|\nabla G^{y}(x)\right| & \leq \frac{C}{r} \sup _{z \in B(x, r / 2)}|G(z, y)| \\
& \leq \frac{C}{r} \sup _{z \in B(x, r / 2)} \frac{C_{k}}{\{1+|z-y| m(V, z)\}^{k}} \frac{1}{|z-y|^{n-2}} \\
& \leq \frac{C_{k}}{\{1+|x-y| m(V, x)\}^{k}} \frac{1}{|x-y|^{n-1}} .
\end{aligned}
$$

On the other hand, if $\delta(x) \leq r / 2$, we observe that $G^{y}=$ $G(\cdot, y)$ satisfies $L G^{y}=0$ in $B(x, \delta(x) / 2)$ for the fixed $y$. Thus by Lemma 15 we have

$$
\left|\nabla G^{y}(x)\right| \leq \frac{C}{\delta(x)} \sup _{z \in B(x, \delta(x) / 2)}|G(z, y)| .
$$

We can choose a point $z^{*} \in B(x, \delta(x) / 2)$ such that

$$
\left|G\left(z^{*}, y\right)\right|=\sup _{z \in B(x, \delta(x) / 2)}|G(z, y)|
$$

and a point $x^{*} \in \partial \Omega$ satisfying $\delta(x)=\left|x-x^{*}\right|$. Then a direct computation implies that

$$
\begin{aligned}
& \delta\left(z^{*}\right) \leq\left|z^{*}-x^{*}\right| \leq \frac{3 \delta(x)}{2}, \\
& \left|z^{*}-y\right| \sim|x-y| \geq 2 \delta(x) .
\end{aligned}
$$

From this and by using Lemma 14, we get that

$$
\begin{aligned}
\left|\nabla G^{y}(x)\right| & \leq \frac{C}{\delta(x)} \frac{C_{k}}{\left\{1+\left|z^{*}-y\right| m\left(V, z^{*}\right)\right\}^{k}} \frac{\delta\left(z^{*}\right)}{\left|z^{*}-y\right|^{n-1}} \\
& \leq \frac{C_{k}}{\{1+|x-y| m(V, x)\}^{k}} \frac{1}{|x-y|^{n-1}},
\end{aligned}
$$

where we have used Lemma 6 in the last inequality. The proof of the lemma is complete.

\section{Local Second-Order Regularity for the Dirichlet Problem}

In this section, we will give some useful a priori $L^{2}$ estimates for the second-order derivative of the solution $u$ to the Dirichlet problem (7), we will show in Theorems 20 and 21 some $L^{2}$ estimates of $\psi \nabla^{2} u$ for the solution $u$ and any smooth function $\psi$. This local second-order regularity will play an important role in the $L^{p}$ regularity argument for the $H_{a t}^{p}$ Dirichlet problem.

Lemma 17. Suppose $\Omega$ is an open domain, $V \in \mathscr{B}_{n}$ and $f \in$ $L^{2}(\Omega)$, then the solution $u \in W_{0}^{1,2}(\Omega)$ of the Dirichlet problem (7) satisfies the following uniform estimate:

$$
\int_{\Omega} V(x)^{2}|u(x)|^{2} d x \leq C \int_{\Omega}|f(x)|^{2} d x,
$$

with the constant $C=C\left(n, \lambda, K_{n}\right)>0$. 
Moreover, for any smooth function $\psi \in C^{\infty}\left(\mathbb{R}^{n}\right)$, the estimate

$$
\begin{aligned}
& \int_{\Omega} V^{2}|u \psi|^{2} d x \\
& \quad \leq C \int_{\Omega}\left(\left|\nabla^{2} \psi\right|^{2}|u|^{2}+|\nabla \psi|^{2}|\nabla u|^{2}+|\psi|^{2}|f|^{2}\right) d x
\end{aligned}
$$

holds with the constant $C=C\left(n, \lambda, K_{n}, M\right)>0$ independent of $\psi$.

Proof. Applying the Green representation formula, the Hölder inequality and Lemma 7, we have that

$$
\begin{aligned}
|u(x)|^{2} & \leq\left|\int_{\Omega} G(x, y) f(y) d y\right|^{2} \\
& \leq \int_{\Omega}|G(x, y)| d y \int_{\Omega}|G(x, y)||f(y)|^{2} d y \\
& \leq C m(V, x)^{-2} \int_{\Omega}|G(x, y)||f(y)|^{2} d y .
\end{aligned}
$$

Hence,

$$
\begin{aligned}
& \int_{\Omega} V(x)^{2}|u(x)|^{2} d x \\
& \leq C \int_{\Omega}\left(\int_{\Omega}|G(x, y)||f(y)|^{2} d y\right) V(x)^{2} m(V, x)^{-2} d x \\
& \quad \leq C \int_{\Omega}|f(y)|^{2}\left(\int_{\Omega} \frac{V(x)^{2} m(V, x)^{-2} d x}{\{1+m(V, x)|x-y|\}^{k}|x-y|^{n-2}}\right) d y \\
& \quad \leq C \int_{\Omega}|f(y)|^{2} d y
\end{aligned}
$$

which is the desired inequality (57).

Noting $u \psi \in W_{0}^{1,2}(\Omega)$ and $\mathscr{L}(u \psi)=-\operatorname{div}(A \nabla \psi) u-$ $2 A \nabla u \nabla \psi+f \psi$, and so by assumption (A2) for the matrix $A$, we can obtain the estimate (58) from the inequality (57). The lemma is proved.

The following a priori estimate is crucial to us.

Lemma 18. Suppose that $\Omega$ is a bounded convex domain or an unbounded region above a convex Lipschitz graph, and $f \in$ $L^{2}(\Omega)$. Let $u \in W_{0}^{1,2}(\Omega)$ be a solution of the Dirichlet problem (7). Then $\psi \nabla^{2} u \in L^{2}(\Omega)$ for any $\psi \in C_{0}^{\infty}\left(\mathbb{R}^{n}\right)$, and one has

$$
\begin{aligned}
\int_{\Omega} \psi^{2}\left|\nabla^{2} u\right|^{2} d x \leq & C \int_{\Omega}\left(|\nabla \psi|^{2}+\psi^{2}\right)|\nabla u|^{2} d x \\
& +C \int_{\Omega}|\psi|^{2}\left(V^{2}|u|^{2}+|f|^{2}\right) d x,
\end{aligned}
$$

with the constant $C=C(n, \lambda, M)>0$.

Proof. Begin by assuming that $\Omega$ is a convex domain with a $C^{2}$ boundary. Let the vector field $W=\psi \nabla u$ and the ball $B$ כ supp $\psi$, then $W \cdot T=0$ for any tangent vector $T$ on the boundary $\partial \Omega$ and $W \equiv 0$ near $\partial B$, so the Kadlec formula on page 134 in [17] implies

$$
\begin{aligned}
\int_{\Omega}(\operatorname{div}(\psi \nabla u))^{2} d x \\
=\sum_{i, j=1}^{n} \int_{\Omega} \frac{\partial W_{i}}{\partial x_{j}} \frac{\partial W_{j}}{\partial x_{i}} d x-\int_{\partial(\Omega \cap B)}(\operatorname{tr} \mathscr{B})(W \cdot N)^{2} d \sigma,
\end{aligned}
$$

where $N$ is the normal vector and $\operatorname{tr} \mathscr{B}$ is the trace of the second fundamental quadratic form on the boundary, that is, the mean curvature of the boundary. For a convex domain we have $\operatorname{tr} \mathscr{B} \leq 0$, and consequently

$$
\sum_{i, j=1}^{n} \int_{\Omega} \frac{\partial W_{i}}{\partial x_{j}} \frac{\partial W_{j}}{\partial x_{i}} d x \leq \int_{\Omega}(\nabla \psi \nabla u+\psi \Delta u)^{2} d x
$$

After a direct computation and using the inequality $2 a b \leq$ $(1 / C) a^{2}+C b^{2}$, we have that

$$
\int_{\Omega} \psi^{2}\left|\nabla^{2} u\right|^{2} d x \leq C \int_{\Omega}|\nabla \psi|^{2}|\nabla u|^{2} d x+2 \int_{\Omega}|\psi|^{2}|\Delta u|^{2} d x,
$$

with the absolute constant $C=C(n)>0$ independent of $\psi$. Thus by the nonsingular change of variables, we get, for any $x_{0} \in \bar{\Omega}$,

$$
\begin{aligned}
\int_{\Omega} \psi^{2}\left|\nabla^{2} u\right|^{2} d x \leq & C \int_{\Omega}|\nabla \psi|^{2}|\nabla u|^{2} d x \\
& +2 \int_{\Omega}|\psi|^{2}\left|\sum_{i, j} \frac{\partial}{\partial x_{i}}\left(a^{i j}\left(x_{0}\right) \frac{\partial u}{\partial x_{j}}\right)\right|^{2} d x,
\end{aligned}
$$

with the absolute constant $C=C(n, \lambda)>0$ independent of $x_{0}$ and $\psi$.

Applying the inequality (65) and using the standard perturbation procedure, we have the absolute constant $C=$ $C(n, \lambda)>0$ such that

$$
\begin{aligned}
& \int_{\Omega} \psi^{2}\left|\nabla^{2} u\right|^{2} d x \\
& \leq C \int_{\Omega}|\nabla \psi|^{2}|\nabla u|^{2} d x+4 \int_{\Omega}|\psi|^{2}|\operatorname{div}(A \nabla u)|^{2} d x \\
& \quad+4 M^{2} \int_{\Omega}|\psi|^{2}\left|x-x_{0}\right|^{2}\left|\nabla^{2} u\right|^{2} d x .
\end{aligned}
$$

Now we decompose $\mathbb{R}^{n}$ into a sequence of cubes $\left\{Q_{k}\right\}$ such that $\mathbb{R}^{n}=\cup Q_{k}, Q_{k}^{o} \cap Q_{i}^{o}=\emptyset$ for any $k \neq i$, and $\operatorname{diam}\left(2 Q_{k}\right)=$ $d<(3 M)^{-1}$ for all $k$. Let the cut-off functions $\chi_{k} \in C_{0}^{\infty}\left(2 Q_{k}\right)$ be the partition of the unity; namely, we can write that $\psi=$ $\sum_{k=1}^{\infty} \psi \chi_{k}$. One can see from the inequality (66) that

$$
\begin{aligned}
\int_{\Omega}\left|\psi \chi_{k}\right|^{2}\left|\nabla^{2} u\right|^{2} d x \leq & C \int_{\Omega}\left|\nabla\left(\psi \chi_{k}\right)\right|^{2}|\nabla u|^{2} d x \\
& +C \int_{\Omega}\left|\psi \chi_{k}\right|^{2}|\operatorname{div}(A \nabla u)|^{2} d x,
\end{aligned}
$$


with the constant $C$ independent of $\psi$ and $k$. Noting that the sequence of cubes $\left\{2 Q_{k}\right\}$ has the finite intersect property, thus there are constants $N>0$ and $C>0$ such that, for every $x \in \Omega$,

$$
\begin{aligned}
& \left(\sum_{k=1}^{\infty}\left|\chi_{k}(x)\right|\right)^{2} \leq N \sum_{k=1}^{\infty}\left|\chi_{k}(x)\right|^{2}, \\
& \sum_{k=1}^{\infty}\left(\left|\chi_{k}(x)\right|^{2}+\left|\nabla \chi_{k}(x)\right|^{2}\right) \leq C .
\end{aligned}
$$

From this and the inequality (67), we can deduce the inequality (61).

A routine limiting argument, see [17] for example, yields the inequality (61) for all convex bounded domains or the unbounded region above a convex Lipschitz graph. The lemma is proved.

We also need the following lemma about the local estimates of the derivatives.

Lemma 19. Suppose that $\Omega$ is a bounded convex domain or an unbounded region above a convex Lipschitz graph, and $f \in$ $L^{2}(\Omega)$. Let $u \in W_{0}^{1,2}(\Omega)$ be a solution of the Dirichlet problem (7). Then, for any $\psi \in C_{0}^{\infty}\left(\mathbb{R}^{n}\right)$, one has

$$
\begin{aligned}
& \int_{\Omega}|\psi|^{2}|\nabla u|^{2} d x+\int_{\Omega} V|\psi|^{2}|u|^{2} d x \\
& \leq C \int_{\Omega}|\nabla \psi|^{2}|u|^{2} d x+C \int_{\Omega}|\psi|^{2}|u f| d x \\
& \int_{\Omega}|\psi|^{2}|\nabla u|^{2} d x+\int_{\Omega} V|\psi|^{2}|u|^{2} d x \\
& \leq C \int_{\Omega}|\nabla \psi|^{2}|u|^{2} d x+C R^{2} \int_{\Omega}|\psi|^{2}|f|^{2} d x
\end{aligned}
$$

with the constant $C=C(n, \lambda)>0$ independent of $\psi$, and $R=$ $\operatorname{diam}(\Omega \cap \operatorname{supp} \psi)$.

Proof. Noting that $u \in W_{0}^{1,2}(\Omega)$ satisfies

$$
\int_{\Omega}\left(A \nabla u \nabla\left(u \psi^{2}\right)+V u^{2} \psi^{2}\right) d x=\int_{\Omega} f u \psi^{2} d x
$$

which, together with the ellipticity of the matrix $A$, follows the inequality (69). Moreover, by this and the Cauchy inequality and the Poincaré inequality, we have that

$$
\begin{aligned}
& \int_{\Omega}|\psi|^{2}|\nabla u|^{2} d x+\int_{\Omega} V|\psi|^{2}|u|^{2} d x \\
& \leq C \int_{\Omega}|\nabla \psi|^{2}|u|^{2} d x+C \int_{\Omega}|\psi|^{2}|u f| d x \\
& \leq C \int_{\Omega}|\nabla \psi|^{2}|u|^{2} d x+\frac{C}{\varepsilon} \int_{\Omega}|\psi|^{2}|f|^{2} d x+\varepsilon \int_{\Omega}|\psi u|^{2} d x \\
& \leq C \int_{\Omega}|\nabla \psi|^{2}|u|^{2} d x+\frac{C}{\varepsilon} \int_{\Omega}|\psi|^{2}|f|^{2} d x+\varepsilon R^{2} \int_{\Omega}|\nabla(\psi u)|^{2} d x .
\end{aligned}
$$

Taking $\varepsilon=(2 R)^{-2}$ in the inequality above, we deduce the inequality (70).

From Lemmas 17-19, we have the following local estimates of the second-order derivatives of the solution $u$ for the Dirichlet problem (7).

Theorem 20. Suppose that $\Omega$ is a bounded convex domain or an unbounded region above a convex Lipschitz graph, $V \in \mathscr{B}_{n}$ and $f \in L^{2}(\Omega)$. Let $u \in W_{0}^{1,2}(\Omega)$ be a solution of Dirichlet problem (7). Then $\psi \nabla^{2} u \in L^{2}(\Omega)$ for any $\psi \in C_{0}^{\infty}\left(\mathbb{R}^{n}\right)$, and one has

$$
\begin{aligned}
& \int_{\Omega}|\psi|^{2}\left|\nabla^{2} u\right|^{2} d x \\
& \leq C \int_{\Omega}\left(\left|\nabla^{2} \psi\right|^{2}+|\nabla \psi|^{2}\right)|u|^{2} d x \\
& \quad+C \int_{\Omega}\left(|\nabla \psi|^{2}+|\psi|^{2}\right)|u f| d x+C \int_{\Omega}|\psi|^{2}|f|^{2} d x \\
& \int_{\Omega}|\psi|^{2}\left|\nabla^{2} u\right|^{2} d x \\
& \leq C \int_{\Omega}\left(\left|\nabla^{2} \psi\right|^{2}+|\nabla \psi|^{2}\right)|u|^{2} d x \\
& \quad+C R^{2} \int_{\Omega}\left(|\nabla \psi|^{2}+|\psi|^{2}\right)|f|^{2} d x+C \int_{\Omega}|\psi|^{2}|f|^{2} d x
\end{aligned}
$$

with the constant $C=C\left(n, \lambda, K_{n}, M\right)>0$ independent of $\psi$, and $R:=\operatorname{diam}(\Omega \cap \operatorname{supp} \psi)$.

Particularly, if $\Omega$ is a bounded convex domain, one has

$$
\int_{\Omega}\left|\nabla^{2} u\right|^{2} d x \leq C(\Omega) \int_{\Omega}|f|^{2} d x
$$

with the constant $C(\Omega)=C\left(n, \lambda, K_{n}, M, \operatorname{diam}(\Omega)\right)>0$.

Proof. Applying Lemmas 17-19, we obtain the inequality (73) from (58), (61), and (69) and get the inequality (74) from (58), (61), and (70). Letting $\psi(x) \equiv 1$ for $x \in \Omega$ in the inequality (74), we obtain the inequality (75).

Theorem 21. Suppose that $\Omega$ is an unbounded region above a convex Lipschitz graph, $V \in \mathscr{B}_{n}$ and $f \in L^{2}(\Omega)$. Let $u \in$ $W_{0}^{1,2}(\Omega)$ be a solution to $-\Delta u+V u=f$ in $\Omega$. Then $\nabla^{2} u \in$ $L^{2}(\Omega)$, and for any $\psi \in C_{0}^{\infty}\left(\mathbb{R}^{n}\right)$, and one has

$$
\begin{aligned}
& \int_{\Omega}|\psi|^{2}\left|\nabla^{2} u\right|^{2} d x \\
& \leq C \int_{\Omega}\left|\nabla^{2} \psi\right|^{2}|u|^{2} d x \\
& \quad+C \int_{\Omega}|\nabla \psi|^{2}|u f| d x+C \int_{\Omega}|\psi|^{2}|f|^{2} d x,
\end{aligned}
$$

with the constant $C=C\left(n, \lambda, K_{n}\right)>0$ independent of $\psi$. Moreover,

$$
\int_{\Omega}\left|\nabla^{2} u\right|^{2} d x \leq C \int_{\Omega}|f|^{2} d x
$$


Proof. Repeating the proof of Theorem 20, and using the inequality (64) in place of the inequality (61), we can then deduce estimate (76). Next, by choosing $\psi=1$ on $B(0, R)$ and $|\nabla \psi| \leq R^{-1},\left|\nabla^{2} \psi\right| \leq R^{-2}$, and by taking the limit as $R$ tends to infinity, we find that $\nabla^{2} u \in L^{2}(\Omega)$ and the global estimate (77) holds.

\section{The Proof of the Main Theorems}

Proof of Theorem 1. By the atomic decomposition theory, it suffices to show the uniform estimates for the solution $u$ of the equation $\mathscr{L} u=a$ in $\Omega$ with the $p$-atom $a$ and $n /(n+1)<$ $p \leq 1$.

Without loss of generality, we assume that $\int_{\Omega} a(x) d x=0$, supp $a \subset Q(0, r) \subset \Omega$ and $\|a\|_{L^{2}} \leq|Q(0, r)|^{1 / 2-1 / p}$, where $Q(0, r)$ denotes the cube with center 0 and side length $r$. Using the inequality (75) of Theorem 20 and the size condition of the atom $a$, we see that, for $n /(n+1)<p \leq 1$,

$$
\int_{\Omega \cap Q(0,2 r)}\left|\nabla^{2} u\right|^{p} d x \leq|Q(0,2 r)|^{1-p / 2}\left(\int_{\Omega}\left|\nabla^{2} u\right|^{2} d x\right)^{p / 2} \leq C,
$$

with the constant $C$ independent of $r$.

Recall the inequality (74) of Theorem 20 and let the cutoff function $\psi \in C_{0}^{\infty}\left(Q\left(0,2^{j+2} r\right) \backslash Q\left(0,2^{j-1} r\right)\right)$ satisfy $\psi=1$ in $Q\left(0,2^{j+1} r\right) \backslash Q\left(0,2^{j} r\right)$ and $|\psi|+2^{j} r|\nabla \psi|+\left(2^{j} r\right)^{2}\left|\nabla^{2} \psi\right| \leq C$ with an absolute constant $C$ independent of $r$ and $j, j=1,2, \ldots$, then we see from the Hölder inequality and the support of the atom $a$ that, for $n /(n+1)<p \leq 1$,

$$
\begin{aligned}
& \int_{\Omega \cap\left(Q\left(0,2^{j+1} r\right) \backslash Q\left(0,2^{j} r\right)\right)}\left|\nabla^{2} u\right|^{p} d x \\
& \leq\left|Q\left(0,2^{j+1} r\right)\right|^{1-p / 2}\left(\int_{\Omega \cap\left(Q\left(0,2^{j+1} r\right) \backslash Q\left(0,2^{j} r\right)\right)}\left|\nabla^{2} u\right|^{2} d x\right)^{p / 2} \\
& \leq C\left(2^{j} r\right)^{n-n p / 2}\left(\left(\left(2^{j} r\right)^{-2}+\left(2^{j} r\right)^{-4}\right)\right. \\
& \left.\times \int_{\Omega \cap\left(Q\left(0,2^{j+2} r\right) \backslash Q\left(0,2^{j-1} r\right)\right)}|u|^{2} d x\right)^{p / 2} \\
& \leq C\left(2^{j} r\right)^{n-n p / 2-2 p}\left(\int_{\Omega \cap\left(Q\left(0,2^{j+2} r\right) \backslash Q\left(0,2^{j-1} r\right)\right)}|u|^{2} d x\right)^{p / 2} .
\end{aligned}
$$

Observing

$$
u(x)=\int_{\Omega}[G(y, x)-G(0, x)] a(y) d y
$$

and using the estimates for the Green function, Lemma 16 and the size condition of the $p$-atom $a$, we can estimate the last integral in the inequality (79) above. Then we have that

$$
\int_{\Omega \cap\left(Q\left(0,2^{j+1} r\right) \backslash Q\left(0,2^{j} r\right)\right)}\left|\nabla^{2} u\right|^{p} d x \leq C\left(2^{j}\right)^{n-p-n p} .
$$

The inequalities (78) and (81) follow that

$$
\int_{\Omega}\left|\nabla^{2} u(x)\right|^{p} d x \leq C+C \sum_{j=1}^{J}\left(2^{j}\right)^{n-p-n p} \leq C,
$$

with the constant $C$ independent of $a$, which implies the theorem.

Proof of Theorem 2. It's enough to consider the case $f(x)=$ $a(x)$, a $p$-atom with its support cube $Q(0, r) \subset \mathbb{R}^{n}$. Then the Hölder inequality and the $L^{2}$-estimate (77) in Theorem 21 give that

$$
\begin{aligned}
& \int_{\Omega \cap Q(0,2 r)}\left|\nabla^{2} u\right|^{p} d x \\
& \quad \leq C|Q(0,2 r)|^{1-p / 2}\left(\int_{\Omega \cap Q(0, r)}|a(x)|^{2} d x\right)^{p / 2} \leq C .
\end{aligned}
$$

On the other hand, if we use the inequality (76) and take the cut-off function $\psi \in C_{0}^{\infty}\left(Q\left(0,2^{j+2} r\right) \backslash Q\left(0,2^{j-1} r\right)\right)$ satisfying $\psi=1$ in $Q\left(0,2^{j+1} r\right) \backslash Q\left(0,2^{j} r\right)$ and $\left(2^{j} r\right)^{2}\left|\nabla^{2} \psi\right| \leq C$ with the constant $C$ independent of $r$ and $j$, then we have

$$
\begin{aligned}
& \int_{\Omega \cap\left(Q\left(0,2^{j+1} r\right) \backslash Q\left(0,2^{j} r\right)\right)}\left|\nabla^{2} u\right|^{2} d x \\
& \quad \leq C\left(2^{j} r\right)^{-4} \int_{\Omega \cap\left(Q\left(0,2^{j+2} r\right) \backslash Q\left(0,2^{j-1} r\right)\right)}|u|^{2} d x .
\end{aligned}
$$

Now using the Hölder inequality and the estimates of the $u$, by the similar arguments as that in the proof of Theorem 1 , we obtain that

$$
\int_{\Omega}\left|\nabla^{2} u(x)\right|^{p} d x \leq C+C \sum_{j=1}^{\infty}\left(2^{j}\right)^{n-p-n p} \leq C
$$

with the constant $C$ independent of the $p$-atom $a$, which implies the theorem.

\section{Conflict of Interests}

The author declares that there is no conflict of interests regarding the publication of this paper.

\section{Acknowledgments}

The work was partly supported by National Natural Science Foundation of China under Grants no. 11171306 and no. 11071065 and funded by Zhejiang provincial Natural Science Foundation of China under Grant no. LY12A01024.

\section{References}

[1] O. A. Ladyzhenskaya, "On some closure of an elliptic operator," Doklady Akademii Nauk, vol. 79, pp. 723-725, 1951 (Russian).

[2] J. Kadlec, "The regularity of the solution of the poisson problem in a domain whose boundary is similar to that of a convex domain," Czechoslovak Mathematical, vol. 14, no. 89, pp. 386393, 1964. 
[3] V. Adolfsson, " $L^{p}$ integrability of second-order derivatives of green potentials in convex do- mains," Pacific Journal of Mathematics, vol. 159, no. 2, pp. 201-225, 1993.

[4] Z. Shen, " $L^{p}$ estimates for Schrödinger operators with certain potentials," Annales de l'institut Fourier, vol. 45, no. 2, pp. 513546, 1995.

[5] S. Sugano, " $L^{p}$ estimates for some Schrödinger type operators and a Calderó-Zygmund operator of Schrödinger type," Tokyo Journal of Mathematics, vol. 30, no. 1, pp. 179-197, 2007.

[6] O. A. Ladyzhenskaya and N. N. Uraltseva, Linear and QuasiLinear Elliptic Equations, Nauka, Moscow, Russia, 2nd edition, 1973, (Russian).

[7] M. Avellaneda and F. H. Lin, " $L^{p}$ bounds on singular integrals in homogenization," Communications on Pure and Applied Mathematics, vol. 44, pp. 897-910, 1991.

[8] K. Kurata and S. Sugano, "A remark on estimates for uniformly elliptic operators on weighted $L^{p}$ spaces and Morrey spaces," Mathematische Nachrichten, vol. 209, pp. 137-150, 2000.

[9] A. Miyachi, " $H^{p}$ space over open subsets of $\mathbb{R}^{n}$," Studia Mathematica, vol. 44, pp. 205-228, 1990.

[10] D. C. Chang, S. G. Krantz, and E. M. Stein, " $H^{p}$ Theory on a smooth domain in $\mathbb{R}^{N}$ and elliptic boundary value problems," Journal of Functional Analysis, vol. 114, no. 2, pp. 286-347, 1993.

[11] X.-X. Tao, "The atomic decompostion for Hardy space on domain and their dual spaces," Journal of Mathematical Study, vol. 29, no. 3, pp. 6-11, 1996.

[12] X.-X. Tao, "The regularity problems with data in Hardy-Sobolev spaces for singular Schrödinger equation in Lipschitz domains," Potential Analysis, vol. 36, no. 3, pp. 405-428, 2012.

[13] X.-X. Tao and H. Wang, "On the Neumann problem for the Schrödinger equations with singular potentials in Lipschitz domains," Canadian Journal of Mathematics, vol. 56, no. 3, pp. 655-672, 2004.

[14] Z. Shen, "On fundamental solutions of generalized Schrödinger operators," Journal of Functional Analysis, vol. 167, no. 2, pp. 521564, 1999.

[15] S.-K. Chua and R. L. Wheeden, "Self-improving properties of inequalities of Poincaré type on s-John domains," Pacific Journal of Mathematics, vol. 250, no. 1, pp. 67-108, 2011.

[16] M. Grüter and K.-O. Widman, "The Green function for uniformly elliptic equations," Manuscripta Mathematica, vol. 37, no. 3, pp. 303-342, 1982.

[17] P. Grisvard, Elliptic Problems in Non-Smooth Domains, Pitman publishing, Boston, Mass, USA, 1985. 


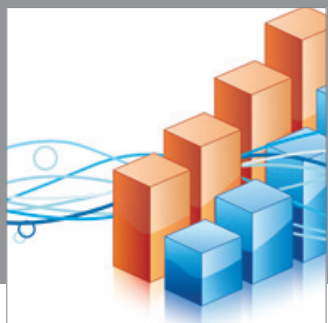

Advances in

Operations Research

mansans

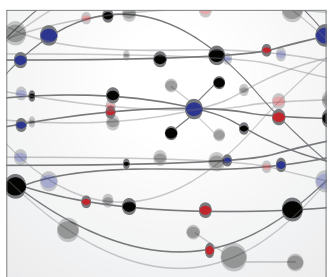

The Scientific World Journal
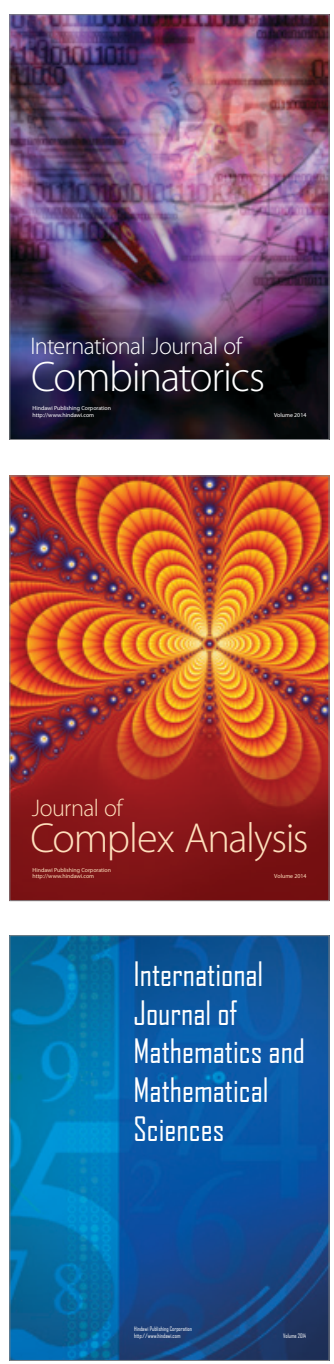
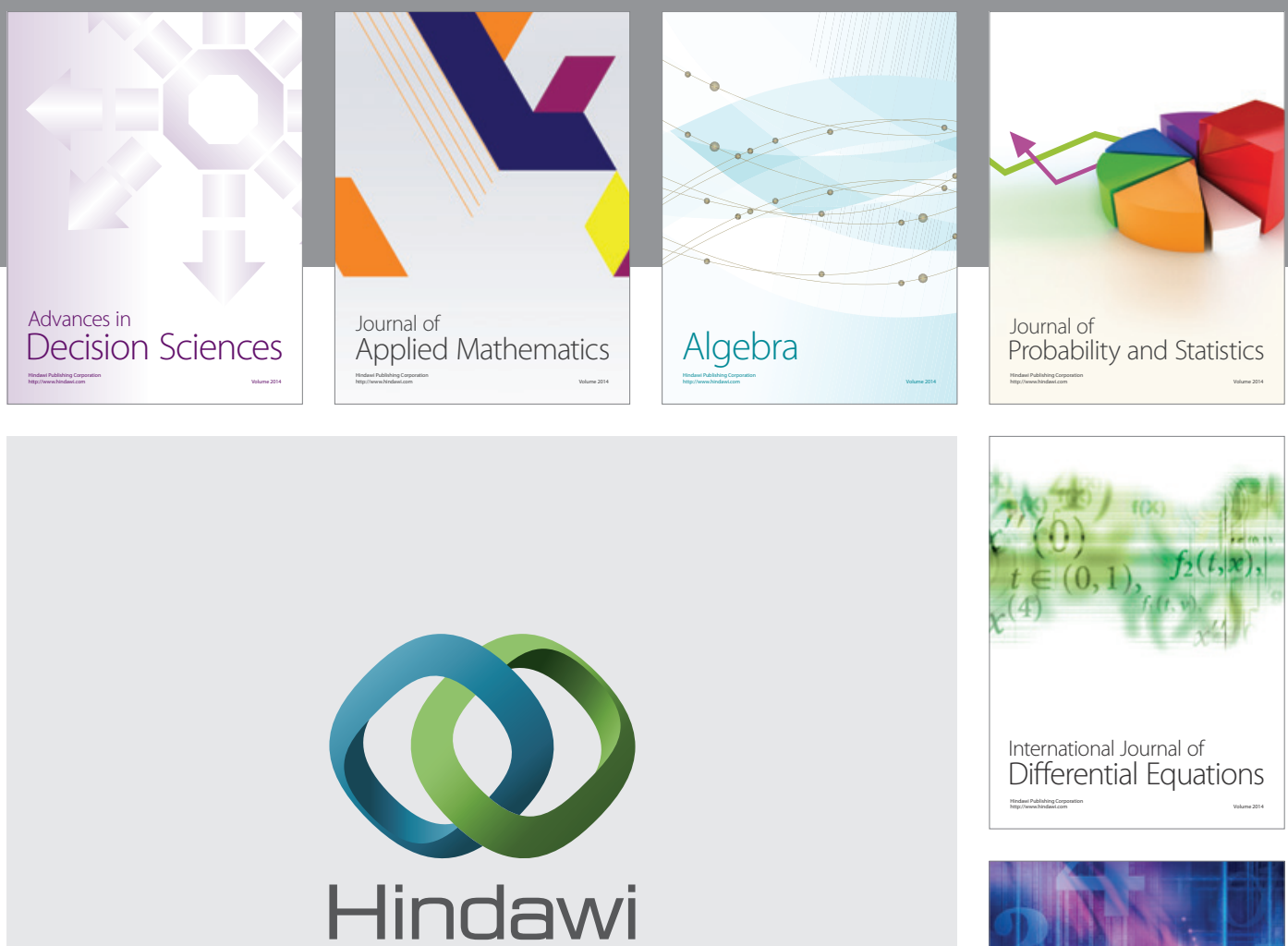

Submit your manuscripts at http://www.hindawi.com
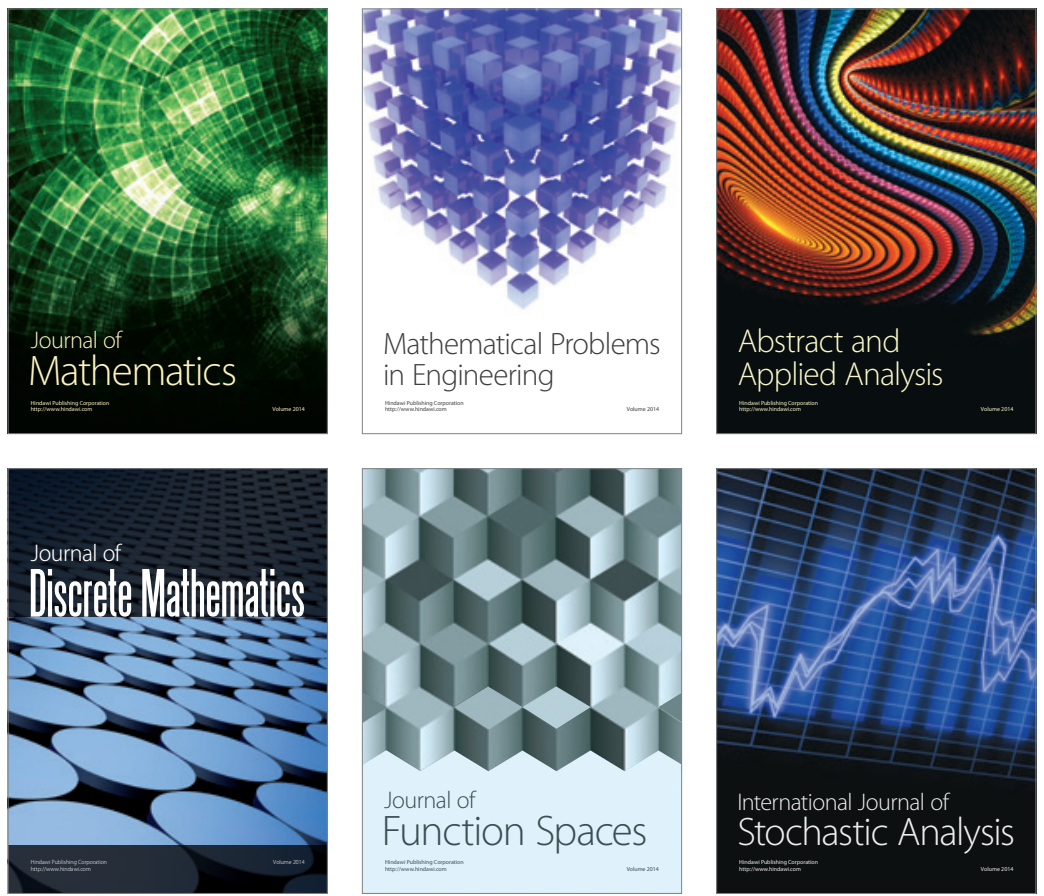

Journal of

Function Spaces

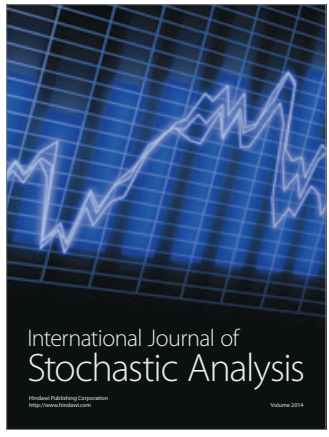

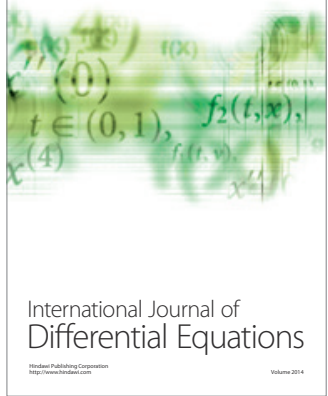
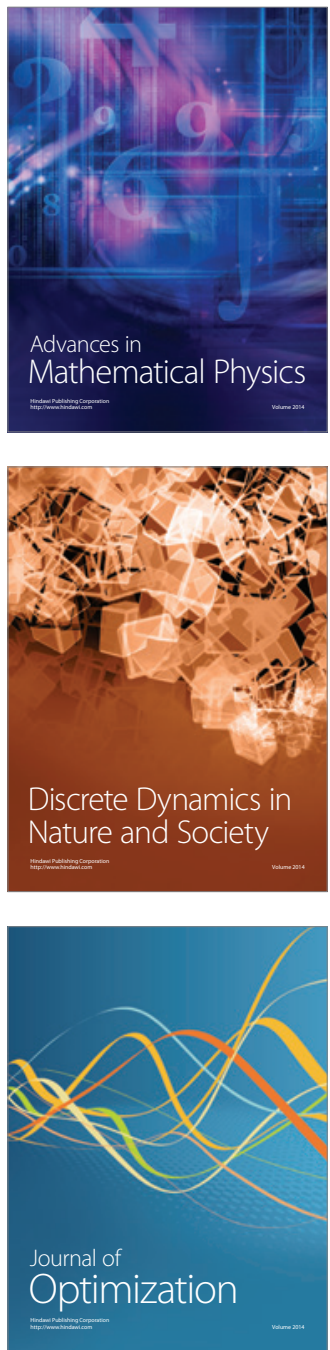